\title{
Hereditary sensory and autonomic neuropathy type 1
}

INSERM

\section{Source}

INSERM. (1999). Orphanet: an online rare disease and orphan drug data base. Hereditary sensory and autonomic neuropathy type 1. ORPHA:36386

Hereditary sensory neuropathy type I (HSN I) is a slowly progressive neurological disorder characterised by prominent predominantly distal sensory loss, autonomic disturbances, autosomal dominant inheritance, and juvenile or adulthood disease onset. 